\title{
Selecting 'interesting' scenarios with which to analyze policy response to potential climate change
}

\author{
Gary W. Yohe \\ Department of Economics, Wesleyan University, Middletown, Connecticut 06457, USA
}

\begin{abstract}
The interactive complexity of the sources and effects of global climate change make it nearly impossible to analyze adaptive and/or abatement response stategies across the full range of possible futures. Researchers are therefore left to investigate their options along a few 'interesting' scenarios of what the future 'might' hold, and so they must focus early attention upon how to select those scenarios. Two selection metrics are examined here. One, based upon minimizing the subjective mean squared error of when some critical state variable (or vector of variables) might cross a response threshold, has its roots in elementary statistical theory; but it is purely a physical measure. A second criterion, based upon minimizing the subjective expected cost of responding at such a threshold, has the advantage of incorporating these costs into the selection process. Subsequent analysis of the potential benefits of abatement along scenarios selected in application of the second metric can thereby incorporate a reflection of efficient adaptation - a first step towards seeing how to strike an appropriate balance between adaptation and abatement.
\end{abstract}

\section{INTRODUCTION}

Most of the issues revolving around possible global, national, and regional response to climate change are enormously complex, interdependent, and shrouded in uncertainty. It must be recognized, therefore, that a clear understanding of the relative merits of the full range of alternative responses to climate change, be they designed to minimize its momentum or to adapt to its consequences, is beyond the pale of simple analytical exercises. Complicated modeling structures are required to properly portray even the current context of potential climate effects. The sheer size of the analytical structures that emerge when these structures are cast forward to portray future effects makes it extraordinarily difficult for any analysis to satisfy simultaneously the need to capture the complexity of the problem and the enormous uncertainty with which the future can be viewed.

Probabilistic portraits of future ranges of important state variables derived from subjective distributions of random control variables cannot, quite simply, be drawn by applying now standard Monte Carlo sampling techniques. [See Andres (1987), Hodges
(1987), Iman \& Conover (1980) and/or Johnson (1987) for description of these techniques; see Nordhaus \& Yohe (1983), Edmonds \& Reilly (1985) and/or Reilly et al. (1987) for coverage of an application to climate change.J The set of possible values for all of the relevant control variables is too large and the requisite modeling structure is too complex. Researchers are left, as a result, with the task of picking a few 'interesting scenarios' upon which to base a few 'what-if' analyses of possible futures; and so they are faced immediately with the fundamental question of how to choose the best, 'most interesting' scenarios. Put succinctly, careful researchers need to ask and answer a question which reads something like 'What criteria should be employed in choosing which scenario(s) to run, and how should those criteria be applied?' A few first steps toward providing a systematic answer to these questions will be advanced here.

\section{A FORMAL REPRESENTATION OF THE PROBLEM}

To set the stage, let future trajectories of some vector of critical state variables be given by $x_{t}(\Theta)$, 
where $\Theta$ is a vector of underlying random variables which drive $\mathrm{x}_{\mathbf{1}}$ into the future. In simpler climate change applications, there may be only one state variable (greenhouse-induced sea level rise, for example), but the underlying random variables could still be numerous and correlated. In other instances (e.g. farm productivity and/or profitability for various alternative crops), even the vector of state variables could be quite involved. In any case, the usual display of the resulting uncertainty depicts a series of subjective distributions $\mathrm{f}_{1}\left(\mathrm{x}_{1} \mid \Theta\right)$, each of which reflects the range of possible values for $\mathbf{x}_{t}$ at some time $t$. Fig. 1 illustrates such a distribution at time $\mathrm{T}$ for a univariate vector $x_{1}$. It provides a vivid picture of the possibilities of future trajectories, but it is not particularly instructive to the analyst who is charged with the task of contemplating when some response to the consequences associated with any one trajectory might be required.

A more productive tack identifies critical threshold values for $\mathbf{x}_{\mathbf{t}}$ which can be associated with clearly discernible impacts and/or the potential need for a well-established adaptive response (Yohe 1991a, b). In a sea level application, such a threshold might be a level of mean spring high tide above which some sort of dike or other protective measure might be required to protect valuable property. Applied to the more complicated agriculture context, a profitability threshold for one crop might signal the likelihood that farmers would switch to some other type of rotation. ${ }^{2}$ Regardless of the context, the identification of a decision-triggering threshold allows the question of representing uncertainty to be rotated by $90^{\circ}$, thereby becoming an issue of anticipating when such a critical threshold might be crossed on the basis of the current information captured in the $\mathrm{f}_{\mathrm{t}}\left(\mathbf{x}_{\mathrm{t}} \mid \Theta\right)$. Fig. 2 shows how that might be accomplished. The distribution of trajectories for $\mathrm{x}_{\mathrm{t}}$ which were supported by $\mathrm{f}_{\mathrm{t}}\left(\mathrm{x}_{\mathrm{t}} \mid \boldsymbol{\Theta}\right)$ are seen there to generate an orthogonal time distribution $\mathrm{g}_{\mathrm{c}}(\mathrm{t} \mid \boldsymbol{\Theta})$ indicating when some threshold value $\mathrm{x}_{\mathrm{c}}$ might be overtaken. ${ }^{2}$

\footnotetext{
${ }^{1}$ See Yohe (1991c) for a discussion of how risk-averse farmers might make such a decision; a suitable threshold could easily emerge from underlying reaction functions based upon subjective views of whether or not the climate had, in fact, changed. Farmers are, after all, equipped only with daily, weekly, monthly, and yearly data on weather - not climate

${ }^{2}$ The precise correspondence between $\mathrm{f}_{t}\left(\mathbf{x}_{t} \mid \Theta\right)$ and $\mathrm{g}_{\mathrm{c}}(t \mid \Theta)$ could be quite complicated, especially in a multivariate application. It is, nonetheless, suggestive of a general procedure which highlights the timing problem and which may best be supported in practice by research directly targeting the $g_{c}(t \mid \Theta)$ distribution (Yohe 1991b)
}

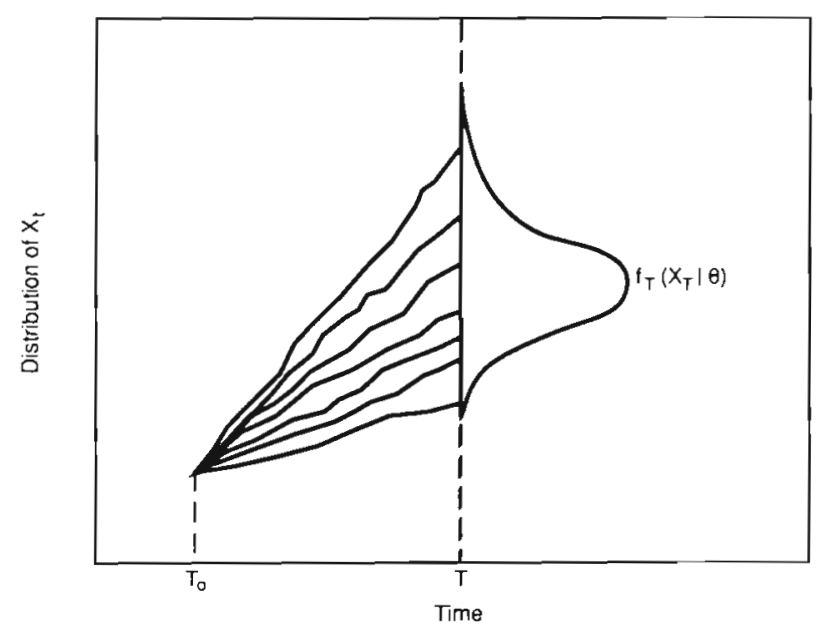

Fig. 1. Any subjective view of the relative likelihood of possible future trajectories for some state variable like $X_{1}$ can be used to produce a corresponding distribution of outcomes, $\mathrm{f}_{\mathrm{T}}\left(\mathrm{X}_{\mathrm{T}} \mid \Theta\right)$ for a particular time $\mathrm{T}$ in the future

The distribution $\mathrm{g}_{\mathrm{c}}(\mathrm{t} \mid \Theta)$ provides direct insight into the full range of times at which a given threshold might be crossed on the basis of current information; and a thorough planning procedure designed to investigate how and when to respond to the impact of crossing that threshold should try to deal with its entire length in some systematic way. Faced with the task of applying enormously complicated systems models to the underlying $\Theta$ to reflect its full dimension, however, planners and researchers alike are left to pick interesting and representative 'what if' scenarios; they are, as a result. confronted with the problem of specifying the criteria upon which to base that selection

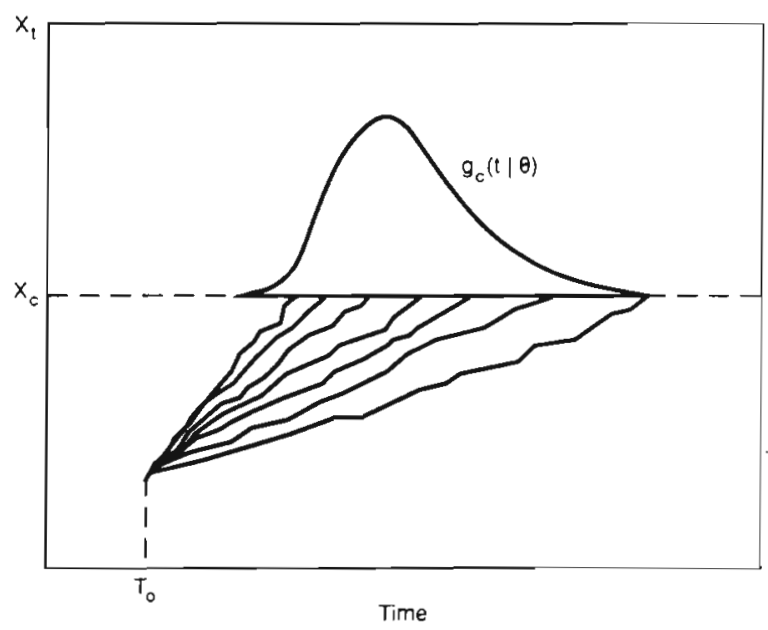

Fig. 2. The same set of possible trajectories for $X_{t}$ displayed in Fig. 1 can produce an orthogonal distribution of future times, $g_{c}(t \mid \Theta)$, at which those trajectories would be expected to cross some predetermined response threshold $\mathrm{X}_{c}$ 


\section{SELECTION CRITERION 1: WEIGHTED MEAN SQUARED ERROR}

One metric upon which to base the selection springs directly from elementary statistical theory: the probabilistically weighted mean squared error (MSE) of anticipating that some critical threshold will be achieved at time $t_{i}$ when, in fact, it will occur at some time $t_{a}$ distributed according to $g_{c}\left(t_{a} \mid \Theta\right)$. This metric is, quite formally, the analog of the estimation error upon which least squared regressions are based; see Johnston (1972) or any elementary statistics text for details. Suppose, more specifically, that $g_{c}\left(t_{d} \mid \Theta\right)$ were a discrete density function. Each cell of $g_{c}\left(t_{a} \mid \Theta\right)$ could then be associated with a threshold crossing time $t_{1}$, and the MSE metric would assign the value

$$
\operatorname{MSE}\left(\mathrm{t}_{1}\right)=\sum_{\mathrm{t}_{\mathrm{a}}}\left\{g_{\mathrm{c}}\left(\mathrm{t}_{\mathrm{a}} \mid \Theta\right)\left(\mathrm{t}_{\mathrm{a}}-\mathrm{t}_{\mathrm{i}}\right)^{2}\right\}
$$

to anticipating crossing the threshold in year $t_{1}$. A best, single guess for the timing of the threshold would therefore be $T$ * which minimized the various $\operatorname{MSE}\left(\mathrm{t}_{\mathrm{i}}\right)$. Specifying a scenario which crossed the threshold at $T^{*}$ could then be advanced as the single best, 'interesting' trajectory according to the MSE metric.

Now suppose that 2 'interesting' trajectories could be considered so that it might prove to be worthwhile to divide the range of threshold-crossing times into a high range and a low range. The underlying subjective density function would not change, but the 'preferred' selection of 'interesting' trajectories certainly could. The initial $\mathrm{T}^{*}$ selected when only one scenario was contemplated might remain as one of the 2 best choices, but it could easily be replaced by 2 entirely different trajectories and associated threshold crossing times.

To see this point, notice that application of the MSE metric would immediately suggest defining 2 subsets of the crossing time range in terms of arriving before or after some benchmark time $t_{c}$. The weighted mean squared error for $t_{i} \leq t_{c}$ would then be

$\operatorname{MSE}_{L}(t)=\left\{\sum_{t_{d} \leq t_{c}} g_{c}\left(t_{a} \mid \Theta\right)\left(t_{a}-t_{i}\right)^{2}\right\} /\left\{\sum_{t_{d} \leq t_{c}} g_{c}\left(t_{a} \mid \Theta\right)\right\}$.

The best choice of scenario given that $t_{a} \leq t_{c}$ would then be $\mathrm{T}^{*} \mathrm{~L}$ which minimized the criterion defined in Eq. (2). A similar computation would also produce $\mathrm{T}^{*}{ }_{\mathrm{H}}$ which minimized the weighted MSE given that $t_{a} \geq t_{c}$. The anticipated error to be expected by running trajectories associated with the pair $\left[\mathrm{T}_{\mathrm{L}}{ }_{\mathrm{i}} \mathrm{T}^{*}{ }_{\mathrm{H}}\right]$ would then simply be the probabilistically weighted sum of $\operatorname{MSE}_{\mathrm{L}}\left(\mathrm{T}^{*} \mathrm{~L}\right)$ and $\mathrm{MSE}_{\mathrm{H}}\left(\mathrm{T}^{*}{ }_{\mathrm{H}}\right)$ :

$$
\begin{aligned}
\operatorname{MSE}_{\mathrm{tc}}=\sum_{\mathrm{t}_{\mathrm{u}} \leq \mathrm{t}_{\mathrm{c}}}\left\{\mathrm{g}_{\mathrm{c}}\left(\mathrm{t}_{\mathrm{a}} \mid \Theta\right)\right\} & \left\{\mathrm{MSE}_{\mathrm{L}}\left(\mathrm{T}^{*} \mathrm{~L}\right)\right\}+ \\
& \sum_{\mathrm{t}_{\mathrm{a}} \leq \mathrm{t}_{\mathrm{c}}}\left\{\mathrm{g}_{\mathrm{c}}\left(\mathrm{t}_{\mathrm{a}} \mid \Theta\right)\right\}\left\{\operatorname{MSE}_{\mathrm{H}}\left(\mathrm{T}^{\cdot}{ }_{\mathrm{H}}\right)\right\} .
\end{aligned}
$$

If the effort involved in running 2 distinct scenarios were worth anything, then the weighted sum computed in Eq. (3) for any $t_{c}$ should be less than the smallest global MSE( $\mathrm{T}^{*}$ ) computed in Eq. (1). Surveying the $\mathrm{MSE}_{\mathrm{tc}}$ across all possible $\mathrm{t}_{\mathrm{c}}$ should, in addition, identify the best pairwise division of the threshold timing range - a most preferred $t_{c^{*}}$ as well as a pair of corresponding best pair of anticipated threshold crossings. In addition, comparing the resulting $\mathrm{MSE}^{*}$ ic with MSE ( $\mathrm{T}^{*}$ ) should provide some insight into whether or not the effort involved in running 2 scenarios would prove to be worthwhile in generating any important, subjectively weighted distinctions across the range of possible futures.

\section{SELECTION CRITERION 2: WEIGHTED LEAST COST OF ADAPTATION}

A second criterion metric, derived in spirit from the MSE but motivated by the fundamental need to investigate the relative merits of responses designed to avert climate change, can now be suggested. The benefit side of any averting policy must be calculated in terms of the cost of prospective damage from the climate change which it prevents, but only after any efficient adaptive reactions that might be forthcoming have been factored into the calculus. An overall efficient response strategy would, in fact, set the marginal cost of all strategies equal to the marginal damage that would nonetheless result from further climate change (Nordhaus 1990). Satisfying these marginal conditions precisely would be extraordinarily difficult, of course, but a first step toward convergence to their satisfaction should consider the relative merits of averting reaction to the threat of climate change in the context of minimum-cost-adaptive reaction to its effects.

Suppose, to formally model this notion, that $\mathrm{C}_{\mathrm{t}}\left(\mathbf{x}_{\mathrm{t}}\right)$ were the stream of expenditures which would be incurred by enacting the least-cost-adaptive response along some trajectory $\mathbf{x}_{\mathrm{t}}{ }^{1}$ Let $\mathrm{C}_{\mathrm{t}}\left(\mathbf{x}_{\mathrm{t}}\right)$, more specifically, reflect the minimum cost of a reaction which could achieve complete adaptation to associated climate

\footnotetext{
'It is implicitly assumed here that decision makers will perfectly identify the trajectory upon which they are riding into the future prior to the need to initiate an adaptive response. This is clearly an extreme assumption, and the concluding section of the paper will suggest how it might be relaxed
} 
change along $\mathbf{x}_{t}$ before some critical threshold $x_{c}$ were crossed. 'The discounted present value of these costs would then be defined by

$$
P V\left\{C_{t}\left(x_{t}\right)\right\}=\sum C_{t}\left(x_{t}\right) /(1+\beta)^{t}
$$

along trajectory $\mathbf{x}_{t}$ given discount rate $\beta$. For the discrete density function $g_{c}\left(t_{a} \mid \Theta\right)$ of Eq. (1), the analogous least expected adaptive cost metric would simply search for the smallest expected cost of responding as if the threshold would be crossed at time $t_{i}$ when it will, in fact, be crossed at time $t_{a}$. Notationally, the single 'most interesting' scenario would be characterized by $T$ * which minimized expected present value:

$$
E P V\left\{C_{t}\left(\mathbf{x}_{\mathbf{t}}\right)\right\}=\sum_{t_{d}}\left\{g_{c}\left(t_{a}(\Theta) \sum C_{t}\left(\mathbf{x}_{t}\right) /(1+\beta)^{l}\right\}\right.
$$

Identifying $\mathrm{T}$ * would, quite clearly, identify a trajectory along which a meaningful comparison with the cost of averting the critical threshold crossing could be conducted; and there is no reason to believe that $\mathrm{T}^{*}=\mathrm{T}^{*} \cdot$

Were it possible to run 2 or perhaps 3 scenarios, then the procedure outlined in the second half of the previous section could produce 2 or 3 such $T$ “ defined in terms of minimum expected adaptive expenditure and could thereby identify 2 or 3 'interesting (adaptation) scenarios' for policy analysis. Consideration of the dual scenario option would, for example, require computing a series of $T_{L} \cdots$ which minimized the analog constrained expected value

$$
\begin{aligned}
& E_{L} P V\left\{C_{t}\left(x_{t}\right)\right\}= \\
& \quad\left[\sum_{t_{a} \leq L_{c}}\left\{g_{c}\left(t_{a} \mid \Theta\right) \sum C_{t}\left(\mathbf{x}_{\mathbf{l}}\right) /(1+\beta)^{\prime}\right\}\right] /\left[\sum_{t_{d} \leq t_{c}} g_{C}\left(t_{a} \mid \Theta\right)\right]
\end{aligned}
$$

for each $t_{c}$ as well as a series of $T_{H}$ " which minimized the corresponding $E_{H} P V\left\{C_{1}\left(x_{1}\right)\right\}$. The best pair $\left\{T_{L} \cdots\right.$, $\left.T_{\mathrm{H}}{ }^{*}\right\}$ would then be determined by the best threshold partition limit $t_{c}{ }^{*}$ which minimized

$\mathrm{EPV}_{\mathrm{tc}}=$

$$
\sum_{t_{d} \leq t_{c}} g_{c}\left(t_{a} \mid \Theta\right) E_{L} P V\left\{C_{1}\left(x_{t}\right)\right\}+\sum_{t_{c}=1} g\left(t_{a} \mid \Theta\right) E_{H} P V\left\{C_{t}\left(x_{t}\right)\right\} .
$$

and 2, adaptive-cost-based, 'interesting' trajectories for $\mathbf{x}_{t}$ would be identified. Again, it is entirely possible that $\mathrm{T}_{\mathrm{L}}{ }^{*} \neq \mathrm{T}_{\mathrm{L}}{ }^{*}, \mathrm{~T}_{\mathrm{H}} \neq \mathrm{T}_{\mathrm{H}}{ }^{*}$, and $\mathrm{t}_{\mathrm{c}}{ }^{*} \neq \mathrm{t}_{\mathrm{c}}{ } \cdot$

The point here is not that the expected cost metric breaks different substantive ground than the MSE metric $_{i}$ it is, instead, that it adds information to the

\footnotetext{
' Residual damages could be included in the computation if adaptation were not complete, but they would only complicate the notation without adding substance to the argument; they are, as a result, ignored here
}

scenario selection process which more fully informs the ultimate purpose of the exercise - investigating the structure of the appropriate mix of policy response decisions. A more thorough discussion of context and import is included in a concluding section, but only after working through a simple illustration which demonstrates that these 2 criteria can easily identify different sets of 'interesting scenarios'.

\section{AN ILLUSTRATIVE EXAMPLE}

Let some state variable $\mathrm{x}_{\mathrm{i}}$ assume an initial value of 1 at time $T_{0}$. Assume that the best current information available with which to contemplate its future values supports only a subjective distribution of annual growth rates that is normal with a mean of $2.0 \%$ per year with a standard deviation of $0.5 \%$ per year. Suppose, further, that 1.2 represents a critical threshold value for $x_{t}$. The variable $x_{t}$ might, for example, reflect an index of the relative frequency of heat and/or water stress in an agricultural region, and a $20 \%$ increase in that index might be considered a trigger for some sort of change in crop, adjustment in crop rotation, or alteration in irrigation practice. If a sea level problem were being studied, then perhaps a $20 \%$ increase in mean spring high tide over current levels might trigger the need for constructing a protective barrier or initiating a program of systematic abandonment. The specifics of the specific climate impact reference are, of course, less important than the identification of a threshold upon which the decision to undertake some adaptive response to the actual trajectory of $x_{t}$ might be based.

A discrete representation of the resulting distribution of the time required for the state variable to grow to the hypothetical threshold value is recorded in the first 2 rows of Table 1 . The remainder of Table 1 shows the expected squared error associated with anticipating that 1.2 would be crossed by $x_{L}$ during the year noted in the leftmost column when, it fact, it would cross the threshold value in the year noted in the first row. The value registered in row (I) of column $(B)$ is, for example, 3.06. It is the value associated with anticipating that $x_{t}=1.2$ in Year 7 when the threshold is crossed in Year 10 and is thus equal to $0.34(10-7)^{2}=3.06$

Table 2 is derived from Table 1, recording the MSE for each possible anticipation under a variety of circumstances. The first row of column (A), for example, records a value of 24.10 . It is the sum of the entries in row (I) of Table 1 as prescribed by Eq. (1), and it represents the mean squared error of anticipating that the 1.2 threshold would be crossed in Year 7 . The first row 
Table 1. Weighted squared errors. Entries represent the expected squared error of anticipating the threshold crossing in the year noted in the left column when, in fact, it occurs in the year given in the first row; they were calculated using Eq. (1). The entries along the diagonal therefore reflect no error because anticipated timing matches actual timing exactly

\begin{tabular}{|c|c|c|c|c|c|c|c|}
\hline \multirow{2}{*}{\multicolumn{2}{|c|}{ Actual year of threshold crossing: }} & (A) & (B) & (C) & (D) & (E) & $(F)$ \\
\hline & & 7 & 10 & 13 & 16 & 19 & 21 \\
\hline & Probability: & 0.28 & 0.34 & 0.27 & 0.08 & 0.02 & 0.01 \\
\hline \multicolumn{8}{|c|}{$\begin{array}{l}\text { Anticipated year of } \\
\text { threshold crossing }\end{array}$} \\
\hline (I) & 7 & 0.00 & 3.06 & 9.72 & 6.48 & 2.88 & 1.96 \\
\hline (II) & 10 & 2.52 & 0.00 & 2.43 & 2.88 & 1.62 & 1.21 \\
\hline (III) & 13 & 10.08 & 3.06 & 0.00 & 0.72 & 0.72 & 0.64 \\
\hline (IV) & 16 & 22.68 & 12.24 & 2.43 & 0.00 & 0.18 & 0.25 \\
\hline (V) & 19 & 40.32 & 27.54 & 9.72 & 0.72 & 0.00 & 0.04 \\
\hline (VI) & 21 & 54.88 & 41.14 & 17.28 & 2.00 & 0.08 & 0.00 \\
\hline
\end{tabular}

Table 2. Weighted squared errors with various differentiating schemes

\begin{tabular}{|c|c|c|c|c|c|c|c|}
\hline \multirow{2}{*}{\multicolumn{2}{|c|}{$\begin{array}{l}\text { Anticipated year of } \\
\text { threshold crossing }\end{array}$}} & \multirow{2}{*}{$\begin{array}{l}(A)^{d} \\
\text { None }\end{array}$} & \multirow{2}{*}{$\begin{array}{c}(B)^{b} \\
\{7,10\} \\
\text { vs rest }\end{array}$} & \multirow{2}{*}{$\begin{array}{l}(\mathrm{C})^{\mathrm{b}} \\
\{7,10,13\} \\
\text { vs rest }\end{array}$} & \multirow{2}{*}{$\begin{array}{c}(D)^{\mathrm{b}} \\
\{19,21\} \\
\text { vs rest }\end{array}$} & \multirow{2}{*}{$\begin{array}{c}(E)^{b} \\
\{7\} \\
\text { vs rest }\end{array}$} & \multirow{2}{*}{$\begin{array}{c}(F)^{\mathrm{b}} \\
\{21\} \\
\text { vs rest }\end{array}$} \\
\hline & & & & & & & \\
\hline (I) & 7 & 24.10 & 4.94 & 14.36 & 19.86 & $0.00^{\circ}$ & 22.36 \\
\hline (II) & 10 & $10.66^{\circ}$ & $4.06^{\circ}$ & $5.56^{\circ}$ & $8.07^{\circ}$ & 11.31 & $9.55^{\circ}$ \\
\hline (III) & 13 & 15.22 & $5.47^{\circ}$ & 14.76 & 14.29 & $7.14^{\circ}$ & 14.73 \\
\hline (IV) & 16 & 37.78 & 7.53 & $3.91^{\circ}$ & 38.51 & 20.97 & 37.91 \\
\hline (V) & 19 & 78.34 & 27.58 & 6.91 & $1.33^{\circ}$ & 52.81 & 79.09 \\
\hline (VI) & 21 & 115.38 & 50.95 & 18.91 & 2.67 & 84.03 & $0.00^{\circ}$ \\
\hline \multicolumn{8}{|c|}{$\begin{array}{l}\text { "Horizontal sum of the row values of Table 1, as prescribed by Eq. (1). The value } 24.10 \text { in row (I) is, for example, the sum } \\
(0.00+3.06+9.72+6.48+2.88+1.96)\end{array}$} \\
\hline \multicolumn{8}{|c|}{$\begin{array}{l}{ }^{b} \text { Weighted sum prescribed by Eq. }(2) \text {; the value } 4.94 \text { in column }(B) \text { of row }(I) \text { is thus the weighted sum }\{(0.00+3.06) / \\
(0.28+0.34)\} \text { because anticipating action in Year } 7 \text { would be considered only when it is known that }\{7,10\} \text { would occur } \\
\text { rather than }\{13,16,19,21\} \text {. Similarly, the value } 50.95 \text { in column }(B) \text { of row (VI) is the weighted sum }(17.28+2.00+ \\
0.08+0.00\} /(0.27+0.08+0.02+0.01)=19.36 / 0.38 \text { because acting in Year } 21 \text { would be anticipated only given } \\
\{13,16,19,21\} \text { rather than }\{7,10\}\end{array}$} \\
\hline \multicolumn{8}{|c|}{ Smallest weighted errors contingent upon the information derived from various benchmarks } \\
\hline
\end{tabular}

of column (B) meanwhile shows a value of 4.94 . This value is the sum of the first 2 entries of row (I) of Table 1 divided by 0.62 (their combined probabilities), as prescribed by Eq. (2), and it represents the mean squared error of anticipating that the threshold would be crossed in Year 7 when only Year 7 and Year 10 are possible. The starred values indicate the smallest weighted errors, contingent upon differentiating across the partitions of the time range indicated at the top of each column.

Table 3 carries the content of Tables 1 and 2 to its conclusion by identifying explicitly the best choices. It shows the expected MSE values computed according to Eq. (3) for each set of best cases identified by the starred entries in Table 2. Notice that any differentiation of scenarios which distinguishes high growth rates from low growth rates along some dividing benchmark is valuable, but some benchmarks are more valuable than others. Distinguishing the heavily weighted 7 yr low extreme from the rest of the distribution would pay off with a $52 \%$ reduction in expected MSE, but distinguishing the 2 even more heavily weighted early possibilities from the rest would pay off the most with a $57 \%$ reduction. On the other side of the distribution, distinguishing the lightly weighted late extreme would not be as worthwhile as distinguishing the latest two; and distinguishing early from late along the arithmetic mean would be worth little more than picking off the upper tail.

An analyst confronted with the choice of running 1 or 2 'interesting scenarios' for $x_{t}$ could, in this simple case, anticipate a potentially significant payoff in minimizing a physical metric of expected error by running 2 scenarios - one which would have been the single best choice crossing the 1.2 threshold in $10 \mathrm{yr}$ (with an annual growth rate of $2.60 \%$ ) and a second crossing 
Table 3. Expected mean squared errors (MSE) for the best choices. Expected MSE is computed according to Eq. (3). The no-differentiation value 10.66 is the starred value in column (A) of Table 2. The next value, 4.57, is the probability weighted average of the starred values in column (B) of Table 2, indicating the weighted squared errors of the best policy: act in Year 10 given $\{7,10\}$ and in Year 13 given $\{13,16,19,21\}$. In particular, $4.57=(0.28+0.34)(4.06)+$ $(0.27+0.08+0.02+0.01)(5.47)$

\begin{tabular}{|c|c|c|}
\hline Circumstance & Best choices & Expected MSE \\
\hline No differentiation & $10 y^{r}$ & 10.66 \\
\hline$\{7,10\}$ vs rest & $\begin{array}{l}7 \text { yr given }\{7,10\} \\
13 \text { yr otherwise }\end{array}$ & 4.57 \\
\hline$\{7,10,13\}$ vs rest & $\begin{array}{l}10 \text { yr given }\{-\} \\
16 \text { yr otherwise }\end{array}$ & 5.38 \\
\hline$\{19,21\}$ vs rest & $\begin{array}{l}19 \text { yr given }\{-\} \\
10 \text { yr otherwise }\end{array}$ & 7.87 \\
\hline$\{7\}$ vs rest & $\begin{array}{c}7 \text { yr given }\{7\} \\
13 \text { yr otherwise }\end{array}$ & 5.14 \\
\hline$\{21\}$ vs rest & $\begin{array}{l}21 \text { yr given }\{21\} \\
10 \text { yr otherwise }\end{array}$ & 9.45 \\
\hline
\end{tabular}

the threshold in $13 \mathrm{yr}$ (with an annual growth rate of $1.4 \%$ ).

Turning now to the expected adaption cost metric, more structure is required. To that end, suppose for the sake of illustration that some secondary analysis of the impact of $x_{1}$ were to support a strategy of initiating a response in anticipation of crossing the $1.2 \mathrm{impact}$ threshold whenever $x_{t}$ crossed a response threshold $\left(\mathrm{x}_{\mathrm{r}}\right)$ of 1.18. Denoting the timing of this response threshold by $t_{r}$, assume as well that an initial fixed cost of $\$ 1$ would be incurred along any $x_{t}$ trajectory at time $\mathrm{t}=\mathrm{t}_{\mathrm{r}}$ and that additional and variable preparation costs of $\$ 1$ would then be proportionately distributed along the time interval between $t_{r}$ and $t_{c}$. Implicit in this illustrative structure, of course, is the notion that the cost of adaptation would be confined to the period between $t_{r}$ and $t_{c i}$ i.e. that ( 1 ) no response and thus no expense would be required prior to $t=t_{r}$ and that (2) no additional response and thus no additional expense would be required after $t=t_{c}$. Recalling the notation of the previous section, then ${ }^{1}$

$$
\begin{array}{ll}
C_{t}\left(x_{1}\right)=0 & \text { for } t<t_{r} \text { or } t>t_{c} i \\
C_{t}\left(x_{1}\right)=1 & \text { when } t<t_{r} ; \\
C_{t}\left(x_{t}\right)=\left(t_{c}-t_{r}\right)^{-1} & \text { for } t_{r}<t \leq t_{c}
\end{array}
$$

\footnotetext{
'This simple structure could easily be extended to include maintenance cost beyond $t_{c}$ but that would again add notational clutter without contributing substance to the illustration
}

The discounted sum of these expenditures would equal

$$
\operatorname{PV}\left\{C_{1}\left(x_{t}\right)\right\}=(1+\beta)^{-t_{T}}+\left(t_{c}-t_{r}\right)^{-1}\left\{\sum_{t_{T}+1}^{t_{r}}(1+\beta)^{\jmath}\right\} .
$$

Finally, assume that even minimal monitoring of $x_{t}$ would constrain the gap between $t_{r}$ and $t_{c}$ to be no less than $1 \mathrm{yr}$ incorrectly anticipating that the critical threshold would be crossed later rather than earlier would result, therefore, in a $\$ 1$ fixed cost expenditure in year $\left(t_{c}-1\right)$ and a maximum variable expense of $\$ 1$ in year $t_{c}$.

The cost portrait depicted in Eq. (5) is extraordinarily simple, but it is not difficult to imagine that its general structure could be the result of an analysis which surveys the range of response options with a selection criterion based upon the current, subjective expected cost of adaptation. It does not recognize that decision makers might adjust $t_{r}$ in response to observed rates of change in $x_{1}$ during the period between $T_{0}$ and Year $\left(t_{c}-1\right)_{i}$ and that is a deficiency. Postponing discussion of how to overcome that deficiency for the moment, it should be noted quickly that a number of important and realistic properties are captured even within its simplicity.

The structure of Eq. (5a) incorporates, first of all, the usual distrinction between fixed, start up costs and ongoing, variable costs; an initial investment is required at time $t_{r}$ to get the response strategy underway, but additional expenditures are also required to bring it to completion. It also reflects, albeit in a simple fashion, the notion that the annual variable costs of response grow as the time available to complete the work shrinks. They climb here on an annual basis as response time shortens solely because they are more concentrated, but that is enough; this negative correlation between expense and available time is a common thread across the investment literature. [See e.g. Chapter 11 in Branson (1972) and cited references: this is a well-established pattern.] Finally, Eq. (5a) reflects a limited degree of contingency reaction by decision makers as the future trajectory of $x$, becomes known. It does not, in particular, assume that no reaction to $x_{t}$ would be forthcoming if the wrong trajectory were anticipated and $x_{r}$ were crossed sooner than expected. It assumes, instead, that it would simply be more costly to respond in that case, and defines a maximum concentration of variable cost by designating 1 yr as the minimum reaction time.

The results of applying the cost metric, based on Eq. (5), are recorded in Tables 4 \& 5. Table 4 shows the discounted cost of responding in anticipation of crossing the 1.2 threshold in the year noted in the leftmost column when, in fact, the threshold would be 
Table 4. Discounted adaptation costs. Computed on the basis of the cost structure of Eq. (5a) and the discounting procedure prescribed in Eq. (5b). When the anticipated year of threshold crossing falls before the actual year, then the fixed cost of responding is incurred when anticipated and the variable cost of preparation is linearly spread over the resulting exagerated time frame When the anticipated year is late, then fixed cost is incurred when $x_{t}$ crosses the initiation tugger (1.18), or 1 yr before crossing the 1.2 threshold, whichever is earlier

\begin{tabular}{|c|c|c|c|c|c|c|c|}
\hline \multirow{2}{*}{\multicolumn{2}{|c|}{ Actual year of crossing threshold: }} & (A) & (B) & (C) & (D) & $(E)$ & $(F)$ \\
\hline & & 7 & 10 & 13 & 16 & 19 & 21 \\
\hline \multicolumn{8}{|c|}{$\begin{array}{l}\text { Anticipated year of } \\
\text { crossing threshold }\end{array}$} \\
\hline (l) & 7 & 1.67 & 0.89 & 0.84 & 0.84 & 0.83 & 0.83 \\
\hline (II) & 10 & 1.67 & 1.53 & 0.81 & 0.77 & 0.77 & 0.77 \\
\hline (III) & 13 & 1.67 & 1.53 & 1.21 & 0.74 & 0.72 & 0.71 \\
\hline (IV) & 16 & 1.67 & 1.53 & 1.40 & 1.35 & 0.68 & 0.66 \\
\hline (v) & 19 & 1.67 & 1.53 & 1.40 & 0.64 & 0.71 & 0.64 \\
\hline (VI) & 21 & 1.67 & 1.53 & 1.40 & 0.75 & 1.23 & 0.66 \\
\hline
\end{tabular}

crossed in the year noted in the first row; a discount rate of $3 \%$ was employed. The figures noted there reflect the effects of 2 possible errors. Being ready to respond too early would, of course, allow the variable cost of adaption to be spread out over a longer period of time, but would impose the fixed cost expenditure too soon. Planning on the basis of late expectations would, on the other hand, run the risk of incurring the more steeply increasing variable costs of accelerated preparation and earlier than expected fixed cost expenditure.

Table 5 summarizes the content of Table 4, much in same way as Table 3 summarized the content of Table 1. The single best scenario selected on the basis of the expected cost of adaption crosses the 1.2 threshold in $7 \mathrm{yr}$ - the shortest time span among the 6 alternatives, and different from the 10 yr horizon that would be selected on the basis of MSE. Constrained to one choice, therefore, the research analyst should note that the costs of being late tend to outweigh the cost of

Table 5. Expected discounted costs for the best choices, computed according to the cost analog of Eq. (3) recorded in Eq. (4d). EPV: expected present value

\begin{tabular}{|lcc|}
\hline Circumstance & Best choices & EPV $\{$ cost $\}$ \\
\hline No differentiation & 7 yr & 1.091 \\
$\{7,10\}$ vs rest & 7 yr given $\{7,10\}$ & 1.177 \\
& 13 yr otherwise & \\
$\{7,10,13\}$ vs rest & 7 yr given $\{7,10,13\}$ & $1.071{ }^{*}$ \\
& 19 yr otherwise & \\
$\{19,21\}$ vs rest & 19 yr given $\{19,20\}$ & 1.087 \\
& 7 yr otherwise & \\
$\{7\}$ vs rest & 7 yr given $\{7\}$ & 1.440 \\
& 13 yr otherwise & \\
$\{21\}$ vs rest & 21 yr given $\{21\}$ & 1.089 \\
& 7 yr otherwise & \\
& &
\end{tabular}

being prepared prematurely and focus on the rapid growth extreme. Given a choice of 2 alternative trajectories, however, the same analyst would look to a threshold division which differentiates across the mean growth rate and add a second, very slow trajectory to the set of 'interesting scenarios.' The best division, in fact, divides the 6 options in half, selecting the $19 \mathrm{yr}$ path with an annual growth rate of $0.96 \%$ to complement the $2.60 \%$ growth of the 7 yr trajectory; these are, as well, different from the best paired selection based on the MSE criterion.

The value of adding a second trajectory is, however, quite small - reducing expected discounted costs by only $2 \%$. The magnitude of this reduction is, of course, specific to the arbitrary nature of the illustration, but a few generalizable insights can be suggested even now. Discounting works to reduce the difference, but that is only part of the story. The cost structure envisioned here incorporates a considerable degree of contingent adaptive response, even when anticipations are incorrect, but still captures a penalty for being ill-prepared. This penalty works against the notion that discounting pushes the efficient timing of adaptive response further into future, opening the question of the value of adaptive learning along any trajectory. Attention can perhaps be profitably diverted from the rapid growth scenarios to the degree that such learning might better inform analysts prior to the need to respond, thereby reducing the penalty for late anticipation of that need and increasing the power of discounting.

It is also interesting to note that differentiation across the threshold timing range does not always reduce the expected cost of adaptation. The constraints on response anticipations imposed by some partitions disallow the best choice of responses in some circumstances, and the resulting second best choices can involve exaggerated expense even in terms of expected discounted value. Finally, the fact 
that 7 and 19 yr crossing times emerge as the best choices for 2 distinct partitions and produce 2 distinct expect cost values is a little anomalous; but this fact, too, can be explained in terms of contrained response selections. Moving the $16 \mathrm{yr}$ response option from one choice set to another does not affect the best choices, but it does alter the set of options best represented by those choices and thus their expected cost of adoption. When scenarios are selected, therefore, care should be taken to recognize explicitly the region of the underlying distribution being reflected by each choice.

\section{CONCLUSIONS}

The criteria suggested here for selecting 'interesting' climate change scenarios are advanced primarily as first steps in confronting a critical methodological challenge. Full-blown probabilistic scenario analyses of what the future might hold may be possible for a limited number of climate-related questions, but their informational and computational requirements will severely limit their applicability when attention is turned to the fundamental issues of response. How should we respond? When should we respond? How should the burden of the cost of restained response and/or the expense of vigorous response be distributed? Answers to questions like these will come only from complex systems approaches which preclude probabilistic analysis. Researchers are thereby left with the initial task of picking a small number of illustrative scenarios and hoping that their selection yields insightful results. This paper highlights the need for developing systematic criteria upon which to base their selections in an effort to improve their chances of making worthwhile choices.

A mean squared error metric is one of the most likely suspects from a statistical perspective, but it is not without its faults. It is, in particular, purely a physical metric which ignores any ranking information which might be gleaned from the ultimate goal of decision-oriented climate change research - finding answers to the questions listed above. Employing, instead, a 'least expected cost of adaptation' metric would show how recognizing this goal might alter the ranking criterion. Economists argue that the best mix of adaptive and averting strategies would malch their marginal costs over time, but how can these costs be compared without fully exercising an elaborate, unifying model which does not yet exist? The least-cost metric proposed here formalizes the notion that the 'interesting' scenarios along which averting responses should be analyzed should be determined by a pro- cess which minimizes the expected cost of adaptation. The first iteration in convergence toward the economists' optimality condition might thereby be completed.

A number of simplifying assumptions were made in the description of the least-cost metric, and their effect on its general applicability should certainly be investigated. It was assumed, first of all, that decision-makers will be able to perceive perfectly the need for adaptation sometime in the future even though they cannot now. This may not be the case, of course, but some future monitoring and/or targeted scientific research will probably improve the information upon which future response decisions will be made. A thorough analyses of the range of response expectations might therefore need to (1) envision the type of information that would be forthcoming along a specific scenario, (2) conceptualize adaptive decisions being made with that information, and (3) weight the costs of those decisions with the current subjective probability that the underlying scenario and its associated experiences will actually occur. (See Yohe (1991a) for a thorough description of how this process might be applied.]

It was also assumed that adaptation would be perfect - that the proposed response to the threat of climate change would totally eliminate any damage that might be forthcoming. This, too, may not be the case. It would not, however, be difficult to include the residual damage of incomplete adaptation in the cost structure. At least conceptually, in fact, the best response along any scenario would match the marginal cost of adapting to the marginal damage nonetheless incurred. There is, as a result, good reason to focus on incomplete adaptation which weighs the diminishing returns of increased adaptive reaction against its increasing marginal cost.

Both of the generalizations noted thus far can be handled with existing and conventional methods. Including their complication may not be worth the effort in a world where uncertainty is sometimes measured in orders of magnitude, but the issues that they raise are quite tractable. Similar reassurances cannot, however, be offered when attention is turned to multidimensional selection processes. Many critical climaterelated state variables will be driven into the future by a large set of random control variables. Crossing a given threshold value at a particular time may not, therefore, be paired uniquely with one combination of control variable trajectories; there may, instead, be an unlimited number of combinations, and a second selection problem arises. Perhaps the probabilistically weighted center of mass should then be selected to drive the representative trajectories, but is the set of possible combinations convex? Perhaps more importantly, is the shape of the trajectory which crosses some 
threshold at some time important? Should the speed of the state variable as it crosses the threshold be considered? These are technical questions, but ones which need to be investigated on, as yet, a case-by-case basis.

A second aspect of multidimensional difficulty is not so daunting. Each climate-related state variable is likely to have one or more critical thresholds for a variety of adaptive responses. Any metric can then be expected to choose an interesting scenario or two for each threshold, so the only question is one of number: what happens if there are too many scenarios? If there is no duplication, reduction in the number of scenarios needs to be based upon a secondary criterion which is consistent with the first. For the mean squared error metric, for example, $n$ scenarios could be chosen for $m \leq n$ different state variables so that they minimize the sum (or weighted sum) of their MSE by starting with the best single choices for each statistical variable and then expanding the set incrementally - adding multiple choice sets for variables whose improved efficiency is greatest. Similar incremental steps, based on reducing total adaptation cost. would accomplish the same task for the cost metric. This is a combination-permutation problem, but tractable once the initial variable specific analyses have been completed

All of these caveats notwithstanding, a fundamental point in the design of climate change decision analysis has been identified here. It is well known that enamel applied to the improperly prepared exterior of a New England house will, in all likelihood, peel after 1 or 2 winters. Homeowners who ignore the proper preparation techniques in their painting projects therefore run a significant risk of facing costly and more involved restoration projects in the not-too-distant future; sanding and scraping 2-yr-old paint is even less welcome than applying a good coat of primer the first time. The same sort of risk may arise in the climate change analysis business if analysts do not pay proper attention to adequate preparation in carefully selecting the 'interesting scenarios' upon which to base their work; they may have to 'undo' the damage of misleading results in the not-too-distant future before they proceed to 'redo' their analysis. The point drawn here is that far too little effort has been expended thus far in defining the rudimentary steps of adequate preparation - defining criteria upon which to select the underlying scenarios

Acknowledgements. Helpful comments during the early stages of this work were provided by Al Liebetrau and Michael Scott of Pacific Northwest Laboratory, Norm Rosenberg and Pierre Crosson of Resources for the Future, Thomas Malone of Sigma Xi and North Carolina State University, and William Nordhaus of Yale University. Funding support was provided by the United States Department of Energy under contract DE-AC06-76RL01830, by Connecticut Sea Grant under R/SL-5 and by the National Science Foundation

\section{LITERATURE CITED}

Andreas, T H. (1987). Statistical sampling strategies. In Proceedings of an Nuclear Energy Agency Workshop. Organization for Economic Cooperation and Development, Paris

Branson, W. (1972). Macroeconomic theory and policy. Harper and Row, New York

Edmonds, J. A., Reilly, J. M. (1985). Global energy: assessing the future. Oxford University Press, New York

Hodges, J. S. (1987). Uncertainty, policy analysis and statistics. Statist. Sci. 2: 259-91

Iman, R. L., Conover, W. J. (1980). Small sample sensitivity analysis techniques for computer models with application to risk assessment. Commun. Statist. B11(3): 311-334

Johnson, M. E. (1987). Multivariate statistical simulation. John Wiley and Sons, New York

Johnston, J. (1972). Econometric methods. McGraw-Hill Book Co., New York

Nordhaus, W. D. (1990). To slow or not to slow. Yale Discussion Paper, New Haven

Nordhaus, W. D., Yohe, G. W. (1983). Future carbon dioxide emissions from fossil fuels. In: Changing climate. Report of Carbon Dioxide Assessment Committee. National Academy Press, Washington, D.C., p. 87-153

Reilly, J. M., Edmonds, J A., Gardner, R. H., Brenkert, A. L (1987). Uncertainty analysis of the IEA/ORAU $\mathrm{CO}_{2}$ emissions model. The Energy Journal 8: 1-29

Yohe, G. (1991a). The cost of not holding back the sea. J Ocean Shoreline Management 15: 233-255

Yohe, G. (1991b). Uncertainty, climate change, and the economic value of information. Policy Sci. 24: 245-269

Yohe, G. (1991c). Imbedding dynamic responses with imperfect information into static portraits of the regional impact of climate change. In: Reilly, J. M. (ed.) Global change: economic issues in agriculture, forestry and natural resources. U.S. Department of Agriculture, Washington, D.C. (in press)

Manuscript first received: May 15, 1991

Revised version accepted: August 14, 1991 